\title{
USO DE UNA RED SOCIAL SEGURA PARA EL ESTUDIO DE LA ANSIEDAD EN LA ADOLESCENCIA
}

\author{
Xavier Bornas, Jordi Llabrés, Maria Balle, Alejandro de la Torre-Luque \\ Y AinA FIOL-VENY \\ Instituto Universitario de Investigación en Ciencias de la Salud (IUNICS), \\ Universidad de las Islas Baleares, Palma de Mallorca, España
}

\begin{abstract}
Resumen: El objetivo del trabajo es presentar brevemente el Proyecto TrANS y los primeros resultados obtenidos a través de dos estudios. Este Proyecto permite el estudio de las trayectorias de la sintomatología ansiosa y la ansiedad-estado en adolescentes mediante el uso de las TIC, en concreto una red social segura para la recogida de datos. En el Estudio 1 se describe la estructura y funcionamiento de la red social segura XS2, además de proporcionarse resultados sobre el acceso y uso de los participantes. En el Estudio 2 se presentan los resultados de las variaciones del estado emocional cotidiano y el uso de estrategias de regulación emocional desadaptativas durante un período de exámenes, así como la influencia de factores de riesgo sobre ellas. Se destacan las ventajas del uso de una red social segura respecto a otros métodos tradicionales de recogida de datos para el estudio de la ansiedad en la adolescencia.
\end{abstract}

Palabras clave: Ansiedad; adolescencia; red social; estado emocional; regulación emocional desadaptativa.

The use of a secure social network for the study of anxiety in adolescence

\begin{abstract}
The aim of this paper is to introduce the TrANS Project and the first results obtained from two studies. This Project allows to examine trajectories of anxiety symptoms and state anxiety in adolescents by means of ICT, in particular a secure social network for data collection. Study 1 describes the structure and functioning of the secure social network XS2 and provides results of the participant's login and use. Study 2 presents the fluctuations of the daily emotional state and the use of maladaptive emotion regulation strategies during an exam period, as well as the influence of risk factors on these fluctuations. In conclusion, the advantages derived from the use of a secure social network are emphasized, in comparison to other traditional data collection methods, in the study of the adolescent anxiety.
\end{abstract}

Keywords: Anxiety; adolescence; social network; emotional state; maladaptive emotion regulation.

\section{INTRODUCCIÓN}

Siendo el objetivo de este monográfico difundir los últimos avances acerca de la aplicabilidad de las tecnologías de la información y la comunicación (TIC) en psicología clínica,

Recibido: 15 junio 2014; aceptado 16 julio 2014.

Este estudio ha sido financiado por el Ministerio de Economía y Competitividad del Gobierno de España (PSI2012-34780).

Correpondencia: Jordi Llabrés, Instituto Universitario de Investigación en Ciencias de la Salud, Universidad de las Islas Baleares. Carretera de Valldemossa km.7.5, 07122 Palma (Mallorca, España). Correo-e: jordi.llabres@uib.cat nos ha parecido oportuno empezar el artículo con una breve descripción del proyecto marco en que se han llevado a cabo los estudios que aquí presentaremos. De este modo el lector podrá ubicar mejor los resultados y tendrá una visión más global del papel de las TIC en lo que estamos investigando.

El proyecto Trayectorias complejas de la ansiedad en la adolescencia: hacia una mejor predicción de la aparición de trastornos de ansiedad (TrAns) pretende obtener conocimiento de las trayectorias que siguen la sintomatología ansiosa y la ansiedad-estado en adolescentes a lo largo de ventanas temporales de 
distinta longitud (siendo la mayor de tres años). Hasta la fecha el estudio de aquellas trayectorias se ha hecho bajo un paradigma lineal que no contempla plenamente la complejidad de las mismas; el proyecto TrAns, en cambio, adopta la Teoría de los Sistemas Dinámicos como marco teórico (o metateórico) idóneo para el estudio del Cambio, por lo que debería ayudar a obtener una visión global de (a) las características de las trayectorias, (b) los aspectos temperamentales y fisiológicos que influyen en las mismas, y (c) la importancia que tienen en relación a aumentos significativos de la sintomatología ansiosa y la eventual aparición de trastornos de ansiedad. Para obtener este conocimiento se ha desarrollado la plataforma digital XS2, con características de red social segura, que sirve para la recogida y gestión de datos autoinformados (y también, en un futuro próximo, para la administración de una intervención psicológica de carácter preventivo). El aspecto más novedoso de dicha plataforma está en el modo de funcionamiento. A diferencia de la mayoría de herramientas existentes (como cuestionarios y tratamientos que se administran a través de Internet) que funcionan de modo individual, ésta funciona, además, de modo social, es decir, los adolescentes de la muestra pueden intercambiar mensajes, chatear, compartir actividades (ya sean lúdicas, informativas, de aprendizaje, etc.). La ventaja de esta herramienta es que el adolescente tiene un papel activo y no se limita a responder preguntas. Gracias a ello se obtiene información menos sesgada y con mayor validez ecológica.

A continuación revisamos la literatura sobre los aspectos clave del proyecto más relacionados con los dos estudios que luego presentaremos: la ansiedad en la adolescencia y el uso de las redes sociales seguras para su estudio. Cabe decir, sin embargo, que al tratarse de un proyecto longitudinal que está en sus inicios, la recogida de datos se irá haciendo en sucesivas oleadas y esperamos publicar en el futuro nuevos resultados.

Los trastornos de ansiedad son las psicopatologías más prevalentes tanto en niños y adolescentes como en personas adultas. La prevalencia en la población infantojuvenil oscila entre un 5\% y un 10\% (Costello, Mustillo, Er- kanli, Keeler, y Angold, 2003; Essau, Conradt, y Petermann, 2000, 2002; Snyder et al., 2009), mientras que a lo largo de la vida se aproxima al 29\% (Kessler, Berglund, Demler, Jin, y Walters, 2005). Según Kessler y sus colaboradores, la incidencia de los trastornos de ansiedad entre los 11 y los 21 años es de un $75 \%$, por lo que la adolescencia constituye un período de gran riesgo para su desarrollo. Una vez iniciado el trastorno, únicamente de un $25 \%$ a un $34 \%$ de niños y adolescentes diagnosticados reciben tratamiento, y de ellos, no todos finalizan la intervención con éxito (p.ej., Essau, 2005; Farrell y Barrett, 2007). Ello contribuye al mantenimiento de alteraciones ansiosas desde la infancia-adolescencia hasta la edad adulta (Costello et al., 2003; Kim-Cohen et al., 2003). Además, la ansiedad infantojuvenil presenta una elevada comorbilidad con diferentes trastornos y constituye un factor de riesgo para otras psicopatologías como la depresión (Cole, Peeke, Martin, Truglio, y Seroczynski, 1998; Craske y Zucker, 2002), interfiriendo notablemente en el funcionamiento personal, familiar, académico y social (p.ej., Costello et al., 2003).

El uso de una perspectiva evolutiva tiene una larga tradición en la investigación en psicopatología, pero el interés por las trayectorias de síntomas infantiles es relativamente reciente en salud mental. Desde esta perspectiva, se intenta identificar grupos de personas que siguen a lo largo del tiempo trayectorias diferentes. Por ejemplo, personas cuyos síntomas muestran una tendencia al aumento frente a aquellas cuyos síntomas se mantienen estables o disminuyen. Una vez que han sido identificadas las trayectorias, pueden establecerse los factores de riesgo o los eventos prospectivos (por ejemplo, el inicio de los trastornos) asociados con dichas trayectorias. Aunque la importancia de este tipo de estudios en la investigación sobre síntomas de ansiedad ha sido reconocida (p.ej. Weems, 2008) los trabajos publicados hasta la fecha son escasos (Bongers, Koot, Van Der Ende, y Verhulst, 2003; Hale, Raaijmakers, Muris, Van Hoof, y Meeus, 2008; van Oort el al. 2009) y los resultados inconsistentes. Algunos estudios longitudinales informan de estimaciones de estabilidad de los trastornos de ansiedad infantiles de entre un 4\% y un $80 \%$ (p. ej., Keller et 
al., 1992; Last et al., 1996; Newman et al., 1996). Esta variabilidad puede ser debida a diferentes motivos, como el tipo de trastorno, el informador, la muestra, el método de evaluación o la cantidad de tiempo transcurrido entre la evaluación inicial y la de seguimiento (ver Silverman y Ollendick, 2005; o Essau y Barrett, 2002, para una revisión). Como decíamos antes, la red social segura XS2, en la que los adolescentes participan de forma activa debería paliar algunos de estos inconvenientes; específicamente los relacionados con la validez de la información que proporciona la muestra.

Entre el bagaje biopsicológico relativamente estable con que cuenta el adolescente en un momento dado y la aparición de un trastorno de ansiedad en un momento posterior, tanto la sintomatología ansiosa (referida a trastornos específicos y a la severidad global) como el nivel cuantitativo de ansiedad-estado (nerviosismo, preocupación, angustia, miedo, etc.) siguen unas trayectorias o cursos temporales que, a día de hoy, son todavía poco conocidos, si bien cabe mencionar la descripción del desarrollo normativo de diversos problemas de conducta a lo largo de la infancia y la adolescencia, publicada por Bongers et al., 2003). A diferencia de los factores temperamentales o de personalidad, que forman parte de aquel bagaje y que han sido extensamente estudiados en las últimas décadas (Anderson y Hope, 2008; Lonigan y Philips, 2001; Muris y Ollendick, 2005), las trayectorias que siguen los síntomas y los niveles de ansiedad sólo han recibido atención en la última década (véase Copeland, Angold, Shanahan, y Costello, 2014). Nuestro proyecto trata de aportar conocimiento sobre una parte al menos de esta laguna.

Cada vez disponemos de más estudios que demuestran que la administración de instrumentos de evaluación psicológica a través de Internet es, en general, tan fiable y válida como sus versiones originales de lápiz y papel (Coles, Cook, y Blake, 2007; Schulenberg y Yutrzenka, 1999; Wyrick y Bond, 2011; Zimmerman y Martinez, 2011). Aunque la evaluación a través de Internet no está exenta de problemas, como puede ser el rechazo de algunas personas al uso de los ordenadores en general, en el caso de los adolescentes, parece que incluso prefieren ser evaluados a través de Internet (Mangunkusumo et al., 2005). Por otra parte, como ocurrió hace algunos años con el término "serious games", recientemente se han comenzado a estudiar los usos no sólo lúdicos de las redes sociales (DeAndrea, Ellison, LaRose, Steinfield, y Fiore, 2012) o las características personales y los motivos psicológicos que hacen que plataformas como Facebook hayan llegado a contar con 500 millones de usuarios (Hew, 2011; Nadkarni y Hofmann, 2012; Ryan y Xenos, 2011). Hasta donde conocemos no se han usado "redes sociales serias" para la evaluación psicológica masiva y longitudinal de población adolescente. En el marco del proyecto TrAns hemos desarrollado una red de este tipo (la ya mencionada XS2) que no sólo permite la administración de cuestionarios estandarizados sino también la evaluación continuada de cualquier variable psicológica, como tendremos ocasión de ver en el Estudio 2. Las características de esta red son las siguientes:

Comunicación segura y anónima. Los participantes se identifican en el sistema a través de unas credenciales y nunca se recoge en la plataforma ningún dato identificativo. Sólo el investigador principal puede acceder a la información que conecte los datos de salud que se registran en la plataforma con los datos personales de identificación (nombre, dirección, teléfono, correo electrónico). La protección de los datos es un objetivo fundamental, y más si cabe al trabajar con menores de edad. Preservando el anonimato se espera también una mayor fiabilidad de los datos. Hay que destacar, sin embargo, que en el momento en que un usuario entre por primera vez en la red ya es "amigo" de todos los usuarios registrados hasta aquel momento, es decir, no necesita ninguna invitación.

Administración eficiente de autoinformes. Al trabajar con muestras grandes es importante poder administrar autoinformes a distancia controlando los datos en todo momento. La plataforma permite controlar las preguntas que no se han respondido, enviar recordatorios a los sujetos sobre autoinformes no contestados, registrar todos los datos obtenidos con marcas de tiempo, exportar los datos fácilmente a software de análisis, etc. 
Psicoeducación individualizada. La plataforma de comunicación segura permite, al estilo del software de e-learning, la orientación individualizada de los adolescentes identificados en grupos de riesgo. Dicho programa, al ser administrado a través de la plataforma, podrá ser interactivo y multimedia.

Fidelización. La plataforma funciona, desde el punto de vista de los participantes, como una red social. Parecida a las que la población adolescente utiliza con asiduidad pero de menor alcance y no sólo con una finalidad lúdica. Para fidelizar a los usuarios e intentar conseguir que se conecten a la plataforma no sólo como obligación de participar en un estudio, se incluyen módulos de mensajería interna para que se puedan comunicar de forma segura, juegos y actividades, información multimedia sobre cuestiones de interés para la adolescencia, etc.

Hecha la introducción en que hemos tratado de justificar tanto el interés del estudio de la ansiedad en la población adolescente como el de hacerlo mediante las tecnologías de la información y la comunicación (TIC) (específicamente mediante la red social segura XS2), presentaremos a continuación dos estudios concretos. El Estudio 1 se centra en la implementación de la red y aporta los datos de participación obtenidos hasta la fecha. Nos ha parecido especialmente oportuno describir el proceso, no exento de dificultades, por el que los diversos institutos de Educación Secundaria Obligatoria (ESO) fueron incorporándose a la plataforma (véase el apartado de Procedimiento del Estudio 1) y describir los datos de participación a modo de resultados. Cabe decir, sin embargo, que la red nos ha facilitado mucha más información (por ejemplo, a través de los intercambios de posts o los diarios personales) que todavía no ha sido convenientemente procesada y que, por ello, no presentaremos en este artículo. El Estudio 2 hizo uso de una de las mejores cualidades de la red XS2: la posibilidad de obtener información diaria sobre el estado psicológico (ánimo, ansiedad, etc.) de los participantes, para ver su relación con otras variables, en este caso, un período de exámenes que podemos considerar como un estresor cotidiano a estas edades, y analizar si la influencia del estresor en el estado diario de los estudiantes venía modulada por factores de riesgo para los trastornos de ansiedad también evaluados a través de la red XS2 mediante cuestionarios.

\section{ESTUDIO 1}

\section{MÉTODO}

\section{Participantes}

Los usuarios de la red XS2 se clasifican en 4 categorías: participantes en el estudio (estudiantes de primero de ESO de 12 a 14 años), supervisores (profesores del centro escolar que pueden acceder a la XS2 e ir revisando si las publicaciones de sus alumnos son adecuadas), investigadores, y administradores (dos investigadores responsables del funcionamiento interno de la red: activación de cuestionarios, gestión de los datos, etc.). La función de los diferentes tipos de usuarios puede verse en la Tabla 1.

Tabla 1. Funciones de los usuarios de la red (señalado con X la función que cada usuario puede llevar a cabo dentro de la XS2)

\begin{tabular}{|c|c|c|c|c|}
\hline & A & I & $\mathrm{S}$ & $\mathrm{P}$ \\
\hline Crear y eliminar usuarios & $X$ & & & \\
\hline $\begin{array}{l}\text { Asignar participantes a los su- } \\
\text { pervisores }\end{array}$ & $\mathrm{X}$ & & & \\
\hline $\begin{array}{l}\text { Compartir publicaciones en la } \\
\text { página de inicio }\end{array}$ & $\mathrm{X}$ & $\mathrm{X}$ & $\mathrm{X}$ & $\mathrm{X}$ \\
\hline $\begin{array}{l}\text { Editar/eliminar publicaciones de } \\
\text { la página de inicio }\end{array}$ & $\mathrm{X}$ & $\mathrm{X}$ & $\mathrm{X}$ & \\
\hline $\begin{array}{l}\text { Enviar/recibir mensajes priva- } \\
\text { dos }\end{array}$ & $\mathrm{X}$ & $\mathrm{X}$ & $\mathrm{X}$ & $\mathrm{X}$ \\
\hline $\begin{array}{l}\text { Ver las respuestas a los cuestio- } \\
\text { narios }\end{array}$ & & $\mathrm{X}$ & & \\
\hline $\begin{array}{l}\text { Comprobar que se han contesta- } \\
\text { do los cuestionarios }\end{array}$ & $\mathrm{X}$ & $\mathrm{X}$ & $\mathrm{X}$ & \\
\hline Contestar los cuestionarios & & & & $\mathrm{X}$ \\
\hline Escribir en el diario personal & & & & $\mathrm{X}$ \\
\hline Ver los diarios personales & & $\mathrm{X}$ & & \\
\hline Destacar publicaciones & $\mathrm{X}$ & & & \\
\hline
\end{tabular}

Nota. $\mathrm{A}=$ Administrador, $\mathrm{I}=$ Investigador, $\mathrm{S}=$ Supervisor, $\mathrm{P}=$ Participante. 
Desde que se activó la red XS2, cuatrocientos cuatro adolescentes (178 hombres, $226 \mathrm{mu}-$ jeres) han sido dados de alta progresivamente a lo largo de cuatro meses. El funcionamiento de la red es similar al de otras redes sociales, pero a esta únicamente pueden acceder aquellos individuos que tienen un código de acceso proporcionado por los investigadores (ver Procedimiento).

\section{Procedimiento}

Se eligieron 12 centros de ESO de Mallorca mediante un muestreo no probabilístico por conveniencia con el objetivo de incluir centros públicos (siete) y centros concertados (cinco), y de zona urbana (siete) y rural (cinco). El proceso de incorporación a la plataforma, descrito a continuación, fue igual para todos los centros. Los pasos seguidos fueron:

(1) Primer contacto telefónico o por correo electrónico con el director o jefe de estudios del centro. En él se describían brevemente los objetivos principales del proyecto y se le ofrecía la oportunidad de participar en el mismo.

(2) Reunión presencial con el equipo directivo del centro escolar para explicar detalladamente los objetivos del proyecto, plantear las ventajas y posibles inconvenientes de su colaboración, mostrar la plataforma y describir el proceso de incorporación al estudio.

(3) Reunión informativa con los tutores de primer curso de ESO, repitiendo la información facilitada al equipo directivo (paso 2), e invitándolos a participar en el estudio como supervisores.

(4) Reunión informativa con los padres de los alumnos de primer curso de ESO.

(5) Distribución y recogida de los consentimientos informados de cada familia interesada en el proyecto. La distribución se realizaba al finalizar la reunión informativa (paso 4) y la recogida a través de los tutores de los alumnos.

(6) Distribución de los códigos de acceso individuales a la red para cada partici- pante. Se describía el estudio a los alumnos durante horario lectivo (habitualmente en horario de tutorías), y se les mostraba la XS2. A aquellos que contaban con el consentimiento informado se les facilitaba el código de acceso y se les explicaban los pasos a seguir para acceder a la red.

(7) Una vez repartidos los códigos, los participantes podían acceder a la XS2 desde cualquier dispositivo con acceso a internet.

A pesar de disponer del código de acceso, muchos alumnos no llegaron a acceder a la red (véase el apartado de Resultados).

\section{Red Social Segura XS2}

Las características de la red han sido descritas en la Introducción y en la Tabla 1 puede verse "quién puede hacer qué" dentro de la red. Los módulos que la componen son los siguientes:

(a) Página de Inicio. Es la primera página que aparece al entrar en la red, un espacio en el que pueden publicar todos los usuarios. Las publicaciones pueden ser en forma de texto, imagen, enlace web y vídeo. También es posible escribir comentarios a las publicaciones hechas por otros usuarios. Junto a cada publicación aparece un botón de denuncia que permite eliminar dicha publicación de la Página de Inicio en caso de que su contenido sea inadecuado (p.ej., contenidos violentos o sexuales).

(b) Página de Perfil. Es un espacio en el que aparecen todas la publicaciones que el usuario ha compartido en la Página de Inicio. Cualquier usuario puede ver la Página de Perfil de otro usuario de la red.

(c) Mensajes. Es un servicio de mensajería interna mediante el cual los usuarios pueden mandarse correos privados entre ellos, sin que lo vean el resto de usuarios.

(d) Comunidad. En éste espacio aparece la lista de todos los usuarios que se encuentran el 
a red, divididos en las cuatro categorías comentadas anteriormente.

(e) Formularios. Los cuestionarios de evaluación se administran a través de este módulo. Los participantes tiene acceso a ellos durante los períodos de evaluación establecidos por los investigadores, y les aparece un aviso con el número de cuestionarios pendientes de responder en su página de Inicio. Una vez respondidos todos, el aviso desaparece. Los administradores gestionan los cuestionarios, activándolos en el momento preciso. La respuesta a los cuestionarios es privada.

(f) Diario Personal. Los participantes pueden escribir en este espacio lo que quieran sobre sus experiencias cotidianas. El espacio es privado y únicamente podrán acceder a su contenido los investigadores, una vez descargados los datos.

(g) Herramientas del Administrador. Este módulo únicamente aparece en la red cuando se accede a ella como Administrador. Desde aquí pueden darse de alta y de baja usuarios y gestionar los cuestionarios. Los administradores también pueden destacar las publicaciones hechas por los usuarios en la página de inicio. Las publicaciones destacadas (cuatro como máximo) aparecen en la parte superior de la Página de Inicio.

\section{RESULTADOS}

Un $25.5 \%$ de los participantes con código de acceso han entrado en la XS2. Los porcentajes de acceso han sido diferentes dependiendo del centro escolar, desde un $10.53 \%$ (centro escolar 10) hasta un 54.74\% (centro escolar 5) (véase la Figura 1).

Desde su activación, el funcionamiento de la XS2 ha ido incrementando progresivamente (véase la Figura 2). Los mensajes privados han sido la herramienta más utilizada, con un máximo de 4.552 mensajes en el último mes del que disponemos de datos, mientras que el diario privado ha sido la menos utilizada, con 132 entradas, también durante ese mismo mes.

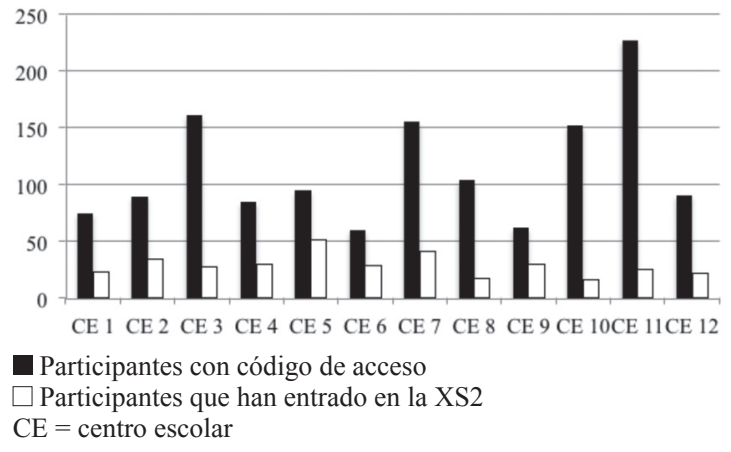

Figura 1. Distribución de participantes por centro escolar.

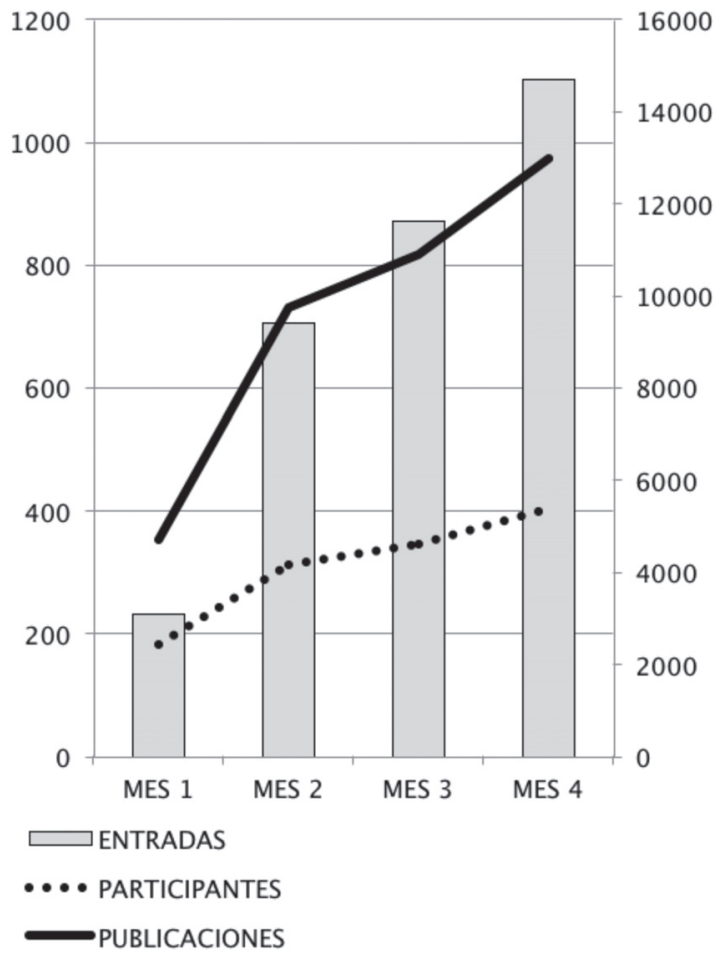

Nota. Tómese el eje vertical primario para Participantes y Publicaciones y el eje vertical secundario para Entradas.

Figura 2. Progresividad en la incorporación de participantes y en la actividad de los mismos en la red XS2.

\section{DISCUSIÓN}

Implementar una red social segura no es fácil. Se trata de un proceso lento debido a todos los pasos previos necesarios para llegar a los adolescentes: desde encontrar centros escolares interesados en el proyecto hasta la recogida de los consentimientos informados de los jóvenes 
y sus padres. Además, la mayoría de usuarios tiene acceso a otras redes sociales con características adicionales, comparado con la XS2. Eso dificulta mantener su interés en esta red. A pesar de ello, como puede verse en los resultados, la actividad de la red ha ido aumentando a lo largo de los meses, y ha permitido la recogida de datos autoinformados sobre estados emocionales, que se presentan en el Estudio 2. De este modo, creemos que la red XS2 es una herramienta útil para estudiar la evolución de los problemas de ansiedad en adolescentes.

\section{ESTUDIO 2}

El estado de ánimo cotidiano se ve influenciado por factores intrínsecos (tanto madurativos y genéticos, como psicológicos) y factores contextuales, si bien puede ser modulado de forma voluntaria con el uso de estrategias de regulación emocional que mejoran la adaptación al contexto. Dicha regulación, sin embargo, puede volverse ineficiente por la acción de ciertos factores psicopatológicos o cuando se debe afrontar una situación especialmente estresante (para una revisión, véase Aldao, Nolen-Hoeksema, y Schweizer, 2010). El periodo de exámenes suele ser considerado como un estresor significativo para gran parte de los adolescentes (Huan, See, Ang, y Har, 2008; Lin y Yusoff, 2013). Este periodo supone enfrentarse a las pruebas de rendimiento para cada materia curricular en un corto de periodo de tiempo, situación percibida como altamente demandante (Lazarus y Folkman, 1984). La carga estresante propia del periodo de exámenes puede llevar a que los adolescentes muestren un importante grado de malestar psicológico $(\mathrm{Pu}-$ twain, 2011; Salmera-Aro y Tynkkinen, 2012).

Los objetivos de este estudio fueron: (a) analizar los cambios en el estado emocional cotidiano (estados de ansiedad y de tristeza) y en el uso de estrategias de regulación emocional desadaptativas (preocupación y evitación conductual) que pueden mostrar los adolescentes a lo largo de un periodo lectivo de exámenes. Asimismo, (b) se pretendía estudiar el efecto de factores de riesgo sobre dichos estados y regulación emocionales. Los factores de riesgo con- siderados fueron: la vivencia reciente de acontecimientos traumáticos, la existencia de sintomatología ansiosodepresiva subclínica, y factores temperamentales asociados al desarrollo de trastornos de ansiedad (alta sensibilidad al castigo y afecto negativo, y bajo control intencional) (véase Hill, Pettit, Lewinsohn, Seeley, y Klein, 2014; Rothbart, 2007; Tortella-Feliu, Balle, y Sesé, 2010).

\section{MÉTODO}

\section{Participantes}

Para preservar la representatividad de la muestra se escogió una submuestra de los participantes en el Estudio $1(N=82,29$ hombres; edad media 12.37 años, $D T=0,57)$. Así, se seleccionaron estudiantes de dos centros de educación secundaria urbanos (uno público, el $26.80 \%$ de la muestra; y uno concertado, el 50\% de la muestra) y uno rural público (23.20\% de la muestra). Un $93.20 \%$ de los participantes tenía hermanos y el $24.10 \%$ vivía con padres separados. Todos los participantes de este estudio se encontraban como usuarios activos en la red XS2 y dieron su consentimiento explícito para participar en el estudio. La presencia de cualquier trastorno de ansiedad fue criterio de exclusión. La participación fue recompensada con premios individuales cuyo valor nunca excedía los 15 euros.

\section{Instrumentos}

Entrevista sociodemográfica para registrar aspectos relacionados con el sexo, la edad y la situación familiar.

Early Adolescent Temperament Questionnaire-Revised (EATQ-R; Ellis y Rothbart, 2001). La traducción y adaptación al catalán fueron realizadas por el Grup de Recerca en Psicopatología Infantojuvenil (GRHCS85) de la Universidad de Gerona. Es un autoinforme para la evaluación del temperamento y comportamiento en niños y adolescentes de 9 a 15 años. Cada ítem se responde mediante una escala de 5 puntos $($ desde $1=$ casi siempre falsa, hasta $5=$ casi 
siempre cierta). Las dimensiones de primer orden que evalúa el instrumento son: control de la activación, control atencional, afiliación, miedo, frustración, placer de alta intensidad; control inhibitorio, timidez, agresión y humor deprimido. Éstas pueden agruparse en cuatro factores de segundo orden: control intencional, surgencia, afecto negativo y capacidad de afiliación. Para este estudio únicamente se tuvieron en cuenta el control intencional y el afecto negativo.

Revised Child Anxiety and Depression Scale (RCADS; Chorpita et al., 2000). Traducido y adaptado por Sandín, Valiente y Chorot (2009). Cuestionario de 47 ítems diseñado para evaluar mediante autoinforme los síntomas DSM-IV de los trastornos de ansiedad y la depresión. Incluye las subescalas: (1) trastorno de ansiedad de separación, (2) fobia social, (3) trastorno de ansiedad generalizada, (4) trastorno de pánico, (5) trastorno obsesivo-compulsivo y (6) trastorno depresivo mayor. El niño/ adolescente debe contestar a los ítems según una escala de frecuencia de $0-3$ puntos $(0=$ nunca, $1=$ a veces, $2=$ a menudo, $3=$ siempre), indicando hasta qué punto reflejan lo que le ocurre al niño/adolescente.

Sensitivity to Punishment and Sensitivity to Reward Questionnaire-Junior (SPSRQ-J; Torrubia, García-Carrillo, Àvila, Caseras, y Grande, 2008). Es un autoinforme adaptado para niños y adolescentes de la versión para adultos desarrollada por Torrubia, Àvila, Moltó, y Caseras (2001). El SPSRQ-J es un cuestionario de 30 ítems de respuesta dicotómica (sí-no) formado por dos escalas de 15 ítems cada una: sensibilidad al castigo y sensibilidad a la recompensa. Las puntuaciones para cada escala se obtienen sumando todas las respuestas afirmativas. Para este estudio únicamente se utilizó la escala de sensibilidad al castigo para el análisis de datos.

Inventario de Acontecimientos Vitales Estresantes (AVE; Oliva, Jiménez, Parra, y Sánchez-Queija, 2008). Autoinforme consistente en un listado de 29 sucesos negativos relativos a sí mismo y a otras personas significativas, que pueden ser experimentados por el adolescente en los contextos familiar, escolar o social (p.ej., divorcio o separación de los padres, muerte de un familiar, repetición de curso, ruptura con la pareja, etc.). Para este estudio se utilizó un único ítem para medir la frecuencia de eventos traumáticos en los últimos seis meses, mediante la pregunta “¿Cuántos de los acontecimientos anteriores te han ocurrido durante los últimos seis meses?". La escala de frecuencia era: 0 , 1-3, 4-6, 7-9, 10-12, 13-15, 16-18, 19-21, 2224, Más de 25.

Escalas visuales analógicas de 100 puntos. Se usaron para evaluar la ansiedad y la tristeza (estados emocionales), y la preocupación y evitación conductual (estrategias de regulación emocional) diarios. Los ítems correspondientes fueron: (a) “¿Cómo me siento?” (desde muy contento -0 puntos- hasta muy triste -100 puntos), (b) “¿Cómo me siento?” (desde muy tranquilo -0 puntos- hasta muy nervioso -100 puntos), (c) “¿Cómo estoy?” (desde no estoy nada preocupado -0 puntos- hasta estoy muy preocupado -100 puntos-), y (d) "Hay cosas y experiencias que pueden causar miedo o nerviosismo (p.ej., subir en ascensor, hablar con un profesor, ver una araña, etc.). Por favor, indica hasta qué punto has dejado de hacer algo o has evitado algo por este miedo o el nerviosismo en el día de HOY" (desde No he dejado de hacer nada -0 puntos- hasta he dejado de hacerlo todo -100 puntos). Los tres primeros ítems fueron adaptados del Inventario de Ansiedad Estado-Rasgo para niños y adolescentes (STAIC, Spielberger, 1989).

\section{Procedimiento}

Se presentó el estudio al consejo escolar y posteriormente se hicieron reuniones con los orientadores de los centros para concretar su desarrollo. Posteriormente, se informó a los participantes sobre el estudio. En este momento, se les pasó la entrevista sociodemográfica.

Tras ello, se pidió a los alumnos que entrasen en la red social XS2 y que completasen los cuestionarios RCADS, EATQ-R y SPSRQ-J, y el ítem sobre los eventos traumáticos recientes. Posteriormente, se solicitó que durante un periodo de 42 días puntuaran su estado emocional y estrategias de afrontamiento dos veces al día, cada día, a través de las escalas visuales analó- 
gicas anteriormente descritas. Así, los participantes debían valorar su estado mediante dichas escalas en horario de mañana (de 7:00 a 11:30) y de tarde (de 15:00 a 20:30). Los registros comenzaron a ser rellenados dos semanas antes del comienzo del periodo de exámenes correspondiente al segundo trimestre del curso 2013/2014. Los adolescentes debían seguir puntuando las escalas durante el periodo de exámenes y las dos semanas siguientes a su finalización.

\section{Análisis de datos}

Para el análisis de datos, se obtuvieron las puntuaciones medias semanales en las escalas analógicas, descartando los valores de los lunes y los viernes por considerarse como transiciones entre los días laborales y fin de semana. Se llevaron a cabo correlaciones bivariadas entre los estados de ansiedad, tristeza, preocupación y evitación, y los factores de riesgo. Se consideraron los factores de riesgo como posibles covariables cuando las correlaciones fueron significativas (Miller y Chapman, 2001).

Para dar respuesta al primer objetivo de este estudio, se realizaron análisis de la covarianza (ANCOVA) de medidas repetidas para cada una de las puntuaciones en las escalas visuales analógicas (variables dependientes) y el momento temporal (con tres niveles: pre-exámenes, exámenes, y post-exámenes) (variable independiente). En caso de que no se cumpliese el supuesto de homocedasticidad, se utilizó la corrección de Greenhouse-Geisser a la hora de considerar el estadístico de contraste.

Para el segundo objetivo, se llevaron a cabo análisis de regresión multivariantes. Se realizaron modelos siguiendo el método de pasos sucesivos con el estado de ansiedad y tristeza, y el uso de la preocupación y la evitación conductual como criterios para cada momento temporal (antes, durante y después del periodo de exámenes). Como predictores, se consideraron el número de acontecimientos traumáticos, la existencia de sintomatología ansiosa y depresiva, y los factores temperamentales evaluados. La introducción de factores en los modelos se basó en las correlaciones significativas obteni- das previamente. Para el cálculo del tamaño del efecto se usó el estadístico eta cuadrado parcial para pruebas comparativas y el coeficiente $R^{2}$ ajustado para modelos regresivos (Cohen, 1988).

Todos los cálculos se realizaron con el paquete estadístico IBM SPSS Statistics, v. 20.

\section{RESULTADOS}

En la Tabla 2 se presentan las puntuaciones medias de los participantes en las diferentes escalas visuales analógicas.

Todos los factores de riesgo correlacionaron con algún estado emocional o estrategia de regulación emocional desadaptativa, excepto el control intencional (véase Tabla 3). Aquellos que mostraron correlaciones significativas fueron utilizados como covariables para los $\mathrm{AN}-$ COVAs descritos a continuación.

Tabla 2. Estados emocionales y uso de estrategias de regulación emocional a lo largo de las fases del estudio

\begin{tabular}{lccc}
\hline & \multicolumn{3}{c}{$M(D T)$} \\
\cline { 2 - 4 } & Pre & Durante & Post \\
\hline Estados emocionales & & & \\
Estado de ansiedad & 15.22 & 15.83 & 15.45 \\
& $(16.69)$ & $(16.20)$ & $(21.04)$ \\
Estado de tristeza & 14.71 & 13.16 & 15.22 \\
& $(23.05)$ & $(20.76)$ & $(20.00)$ \\
\hline Estrategias de RE desadaptativas & & \\
Preocupación & 14.10 & 16.41 & 14.82 \\
& $(16.79)$ & $(17.20)$ & $(16.87)$ \\
\multirow{2}{*}{ Evitación conductual } & 10.98 & 8.02 & 7.60 \\
& $(17.93)$ & $(15.44)$ & $(14.06)$ \\
\hline
\end{tabular}

Nota $. \mathrm{RE}=$ regulación emocional

A partir de los resultados obtenidos, no se observaron fluctuaciones en los estados emocionales evaluados en este estudio, ni para ansiedad ni para tristeza $(p>0,05)$. Sin embargo, sí que se encontraron fluctuaciones en el uso de las estrategias de regulación emocional a lo largo de las fases del estudio, tanto en el uso de la preocupación, $F(2,64)=3.54, p<.05 ; \eta_{\mathrm{p}}^{2}$ $=.10$; como en el uso de la evitación conductual, $\operatorname{con} F(1,35,47,14)=3.44, p<.05 ; \eta_{\mathrm{p}}^{2}=$ .10. Se observó un mayor uso de la preocupa- 
Tabla 3. Correlaciones de Pearson entre los factores de riesgo y los estados emocionales y estrategias de regulación emocional desadaptativas

\begin{tabular}{|c|c|c|c|c|c|c|c|c|c|c|c|c|}
\hline & \multicolumn{6}{|c|}{ Estados emocionales } & \multicolumn{6}{|c|}{ Estrategias de RE desadaptativas } \\
\hline & \multicolumn{3}{|c|}{ Ansiedad estado } & \multicolumn{3}{|c|}{ Tristeza estado } & \multicolumn{3}{|c|}{ Preocupación } & \multicolumn{3}{|c|}{ Evitación conductual } \\
\hline & Pre & Durante & Post & Pre & Durante & Post & Pre & Durante & Post & Pre & Durante & Post \\
\hline \multicolumn{13}{|l|}{ Factores de riesgo } \\
\hline $\begin{array}{l}\text { Acontecimientos traumáticos } \\
\text { recientes }\end{array}$ & $0.31^{*}$ & 0.04 & 0.22 & $0.35^{* *}$ & 0.21 & 0.23 & $0.30^{*}$ & 0.15 & 0.20 & $0.47 * *$ & -0.02 & 0.10 \\
\hline Sintomatología ansiosa & $0.38^{* *}$ & $0.40^{* *}$ & 0.24 & $0.31^{*}$ & 0.24 & 0.15 & $0.37 * *$ & $0.34^{*}$ & 0.26 & $0.32 * *$ & $0.32 *$ & 0.21 \\
\hline Sintomatología depresiva & $0.41^{* *}$ & 0.18 & 0.13 & $0.39 * *$ & 0.17 & 0.16 & $0.49 * *$ & 0.17 & $0.31^{*}$ & $0.37 * *$ & 0.15 & 0.10 \\
\hline Sensibilidad al castigo & $0.40^{* *}$ & $0.36^{* *}$ & 0.19 & 0.23 & 0.22 & 0.25 & $0.46^{* *}$ & $0.32 *$ & $0.37^{*}$ & 0.23 & $0.30 *$ & 0.22 \\
\hline Afecto negativo & -0.11 & -0.17 & -0.08 & -0.19 & -0.18 & $-0.35^{*}$ & -0.16 & -0.22 & -0.21 & -0.20 & -0.12 & -0.19 \\
\hline Control intencional & 0.16 & 0.01 & 0.01 & 0.12 & 0.14 & 0.04 & 0.26 & 0.06 & 0.17 & -0.03 & 0.01 & 0.09 \\
\hline
\end{tabular}

Nota. $\mathrm{RE}=$ regulación emocional

$* p<.05 ; * * p<.01$

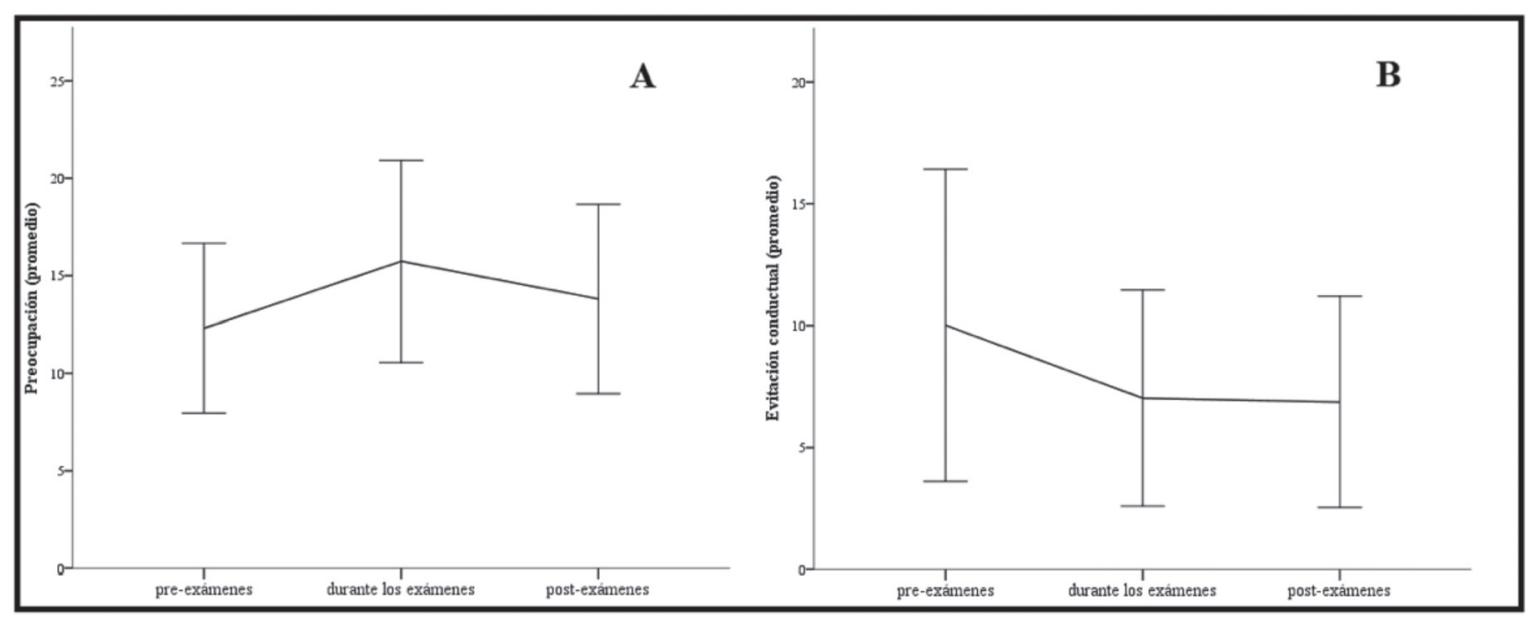

Nota. En la Figura A se observa cómo fluctúa el uso de la preocupación cuando el adolescente debe enfrentarse al periodo de evaluaciones de exámenes. En la Figura B se presenta el uso de la evitación bajo las mismas circunstancias. El eje y ha sido adaptado a los valores reales obtenidos. Para ambos gráficos, las barras de error se han construido a partir del intervalo de confianza al $95 \%$.

Figura 3. Uso de las estrategias de regulación emocional desadaptativas a lo largo de las fases del estudio.

ción en el periodo de exámenes, mientras que la evitación fue más usada en las semanas previas al comienzo de dicho periodo (véase la Figura 3). Además, se encontró un efecto de interacción del uso de la evitación con la vivencia de acontecimientos traumáticos $(F(1,35$, $\left.47,14)=7.49, p<.01 ; \eta_{p}^{2}=.18\right)$. Dicho efecto de interacción venía a señalar que los participantes que recientemente habían vivido más eventos traumáticos utilizaban más frecuentemente la evitación en el periodo previo a los exámenes.

Los factores de riesgo que correlacionaron significativamente con los estados emocionales y de regulación emocional cotidianos fueron utilizados como predictores en los análisis de regresión (véase la Tabla 3).

Los análisis de regresión revelaron que la sensibilidad al castigo era un predictor significativo $(\beta=.40 ; t=2.98 ; p<.001)$ del estado de ansiedad cotidiano pre-exámenes $\left(R_{\mathrm{AJ}}^{2}=.12\right)$. En este momento concreto, la sintomatología ansiosa subclínica $(\beta=.42 ; t=3.60 ; p<.001)$ predijo el nivel de preocupación diario $\left(R_{\mathrm{AJ}}^{2}=\right.$ .16). Finalmente, la sintomatología depresiva subclínica $(\beta=.37 ; t=3.24 ; p<.01)$ y la vivencia de acontecimientos traumáticos $(\beta=.23 ; t$ $=2.00 ; p<.05$ ) explicaron un porcentaje signi- 
ficativo de la evitación cotidiana $\left(R_{\mathrm{AJ}}^{2}=.25\right)$, de nuevo antes de los exámenes. No se observó ningún modelo explicativo significativo para el estado de tristeza. Tampoco se encontraron otros modelos para cualquiera de estos criterios durante el periodo de exámenes y posterior.

\section{DISCUSIÓN}

En este estudio se pretendía analizar cómo variaba el estado y el uso de estrategias de regulación emocional ante un estresor cotidiano, como es el periodo de exámenes. Asimismo, se pretendía estudiar la influencia de factores de riesgo para trastornos de ansiedad sobre estas variables, antes, durante y después de los exámenes. Aunque no se observaron fluctuaciones significativas entre fases para los estados emocionales, el uso de la preocupación y la evitación conductual sí varió a lo largo de las fases. Concretamente, se observó que los participantes usaban más frecuentemente la evitación conductual en el periodo pre-exámenes; y la preocupación, en cambio, era más usada durante las semanas de exámenes. La evitación parece asociarse a la anticipación de emociones negativas como ansiedad o ira (Schutz, Benson, y Decuir-Gunby, 2008). De este modo, los participantes tenderían a evitar el afrontamiento de situaciones demandantes que suelen llevar a mejor rendimiento académico (ej.: ir al despacho del profesor a resolver dudas o quedar con compañeros para que expliquen conceptos complejos, etc.). Por otro lado, en el periodo de exámenes per se, la preocupación podría ser utilizada como una estrategia para reducir, a corto plazo, las consecuencias aversivas derivadas del estresor y anticipar posibles pautas de actuación, aunque otras veces haya servido para focalizar la atención ante el supuesto fracaso propio de suspender el examen (Bodas, Ollendick, y Sovani, 2008; Borkovec, Pruzinsky, y DePree, 1983; Brosschot, Verkuil, y Thayer, 2010).

En relación al segundo objetivo, los resultados encontrados muestran que, si bien los factores de riesgo presentaban asociaciones significativas con los criterios para todos los periodos, sólo se destacaron como predictores en la etapa de ansiedad anticipatoria. El periodo de exámenes se considera un estresor con su perfil característico (Lazarus y Folkman, 1984); esto es, se observa un periodo de ansiedad anticipatoria (periodo pre-exámenes) y periodo de máxima ansiedad (periodo de exámenes); posteriormente, se encontraría la fase de espera de notas (post-exámenes). En el periodo pre-exámenes los individuos adaptan su patrón conductual para hacer frente al estresor (Zeidner, 1998), en este caso, de forma desadaptativa, y con una mayor influencia de los factores de riesgo. Debido a ello, sería interesante promover el uso de estrategias de regulación emocional adaptativas, especialmente durante este periodo.

\section{DISCUSIÓN GENERAL}

En este artículo hemos presentado brevemente el proyecto marco TrAns, que pretende obtener conocimiento de las trayectorias que siguen la sintomatología ansiosa y la ansiedad-estado en adolescentes, a lo largo de ventanas temporales de distinta longitud, mediante una red social segura desarrollada para tal fin (la red XS2). Es en este marco donde se ubican los dos estudios expuestos. El primero describe la implementación de la red, que cuenta con más de cuatrocientos participantes activos repartidos en doce institutos de ESO. Quizá lo más destacable es la enorme inversión de tiempo y esfuerzo necesaria para llegar a incorporar a los participantes en la red y para mantenerlos ahí. Contactos telefónicos, visitas, reuniones con diversos órganos y personas (supervisores y equipos directivos) de cada instituto, información a los estudiantes y a los padres y madres, obtención de los consentimientos informados, reparto de códigos, seguimiento de los registros iniciales de usuarios, dinamización de la red, etc. Todo ello lleva inevitablemente a preguntarse si realmente vale la pena, siendo, finalmente, la respuesta afirmativa. Los estudios longitudinales sobre ansiedad infantojuvenil son, a día de hoy, aún escasos. Las limitaciones relativas a la recogida de datos tradicional durante varios años, como cambios de colegio o la ausencia de algunos alumnos durante las 
sesiones de evaluación de papel y lápiz (p.ej., Sportel, Nauta, de Hullu, y de Jong, 2013), son superables mediante la evaluación electrónica. Además, dicho sistema permite una gestión de los datos más segura y eficiente, optimizando el manejo de gran cantidad de información. En este sentido, también facilita la recogida de datos diariamente, integrando así diferentes niveles de evaluación (años-meses-días) mediante una misma herramienta. El formato de red social puede ayudar, además, a solucionar los problemas de adherencia inherentes a cualquier estudio longitudinal, ya que permitirá mantener la motivación del adolescente a participar en el estudio durante un largo período de tiempo.

El segundo estudio es una primera demostración de la utilidad de la red XS2 para llevar a cabo investigaciones convencionales sobre el tema de la ansiedad en la adolescencia. En este sentido, más allá de los resultados que ya hemos discutido anteriormente, queremos señalar la flexibilidad de la red como instrumento de estudio. La red XS2 permite incorporar fácilmente preguntas (sobre estados o estrategias, como hemos visto, pero también sobre cualquier otra variable psicológica) que en cada momento se consideren clave para un estudio específico. Además, como mencionamos anteriormente, la información que recogemos no es sólo reactiva (cuestionarios o preguntas): es también proactiva y esto es una ventaja indudable de la red. Los estudiantes envían sus posts, cuelgan materiales, indican links, comentan lo que ocurre, escriben en sus diarios personales, etc., proporcionando una ingente cantidad de información no reactiva (menos sesgada en este sentido) cuyo análisis será de gran utilidad, sin duda, para avanzar en el conocimiento de los problemas de ansiedad en esta fase crucial del desarrollo humano que es la adolescencia.

\section{REFERENCIAS}

Aldao, A., Nolen-Hoeksema, S., \& Schweizer, S. (2010). Emotion-regulation strategies across psychopathology: A meta-analytic review. Clinical Psychology Review, 30, 217-237.

Anderson, E.R., \& Hope, D.A. (2008). A review of the tripartite model for understanding the link between anxiety and depression in youth. Clinical Psychology Review, 28, 275-287.

Bodas, J., Ollendick, T.H., \& Sovani, A.V. (2008). Test anxiety in Indian children: a cross cultural perspective. Anxiety, Stress, \& Coping: An International Journal, 21, 387-404.

Bongers, I.L., Koot, H.M., van der Ende, J., \& Verhulst, F.C. (2003). The normative development of child and adolescent problem behavior. Journal of Abnormal Psychology, 112, 179-192.

Borkovec, T.D., Robinson, E., Pruzinsky, T., \& DePree, J.A. (1983). Preliminary exploration of worry: Some characteristics and processes. Behaviour Research \& Therapy, 21, 9-16.

Brosschot, J.F., Verkuil, B., \& Thayer, J.F. (2010). Conscious and unconscious perseverative cognition: Is a large part of prolonged physiological activity due to unconscious stress? Journal of Psychosomatic Research, 69, 407-416.

Cohen, J. (1988). Statistical power analysis for the behavioral sciences (2nd ed.). Mahwah: Lawrence Erlbaum.

Cole, D.A., Peeke, L.G., Martin, J.M., Truglio, R., \& Seroczynski, A.D. (1998). A longitudinal look at the relation between depression and anxiety in children and adolescents. Journal of Consulting and Clinical Psychology, 66, 451-460.

Coles, M.E., Cook, L.M., \& Blake, T.R. (2007). Assessing obsessive compulsive symptoms and cognitions on the internet: Evidence for the comparability of paper and Internet administration. Behaviour Research and Therapy, 45, 2232-2240.

Copeland, W.E., Angold, A., Shanahan, L., \& Costello, E.J. (2014). Longitudinal patterns of anxiety from childhood to adulthood: The Great Smoky Mountains Study. Journal of American Academy of Child and Adolescent Psychiatry, 53, 21-33.

Costello, E.J., Mustillo, S., Erkanli, A., Keeler, G., \& Angold, A. (2003). Prevalence and development of psychiatric disorders in childhood and adolescence. Archives of General Psychiatry, 60, 837-844.

Craske, M.G., \& Zucker, B.G. (2002). Prevention of anxiety disorders: A model for intervention. Applied and Preventive Psychology, 10, 155-175.

DeAndrea, D.C., Ellison, N., LaRose, R., Steinfeld, C., \& Fiore, A. (2012). Serious social media: On the use of social media for improving students' adjustment to college. The Internet and Higher Education, 15, 15-23.

Essau, C.A. (2005). Frequency and patterns of mental health services utilization among adolescents with anxiety and depressive disorders. Depression and Anxiety, 22, 130-137.

Essau, C.A.. \& Barrett, P. (2002). Developmental issues in the assessment of anxiety. En C. A. Essau y F. Peterman (Eds.), Anxiety disorders in children and 
adolescents: Epidemiology, risk factors and treatment (pp. 75-110). Nueva York: Taylor \& Francis.

Essau, C.A., Conradt, J., \& Petermann, F. (2000). Frequency, comorbidity, and psychosocial impairment of specific phobia in adolescents. Journal of Clinical Child Psychology, 29, 221-231.

Essau, C.A., Conradt, J., \& Petermann, F. (2002). Course and outcome of anxiety disorders in adolescents. Journal of Anxiety Disorders, 16, 67-81.

Farrell, L.J., \& Barrett, P.M. (2007). Prevention of childhood emotional disorders: Reducing the burden of suffering associated with anxiety and depression. Child and Adolescent Mental Health, 12, 58-65.

Hale, W.W., Raaijmakers, Q., Muris, P., van Hoof, A., \& Meeus, W. (2008). Developmental trajectories of adolescent anxiety disorder symptoms: A 5-year prospective community study. Journal of the American Academy of Child and Adolescent Psychiatry, 47, 556564.

Hew, K.F. (2011). Students' and teachers' use of Facebook. Computers in Human Behavior, 27, 662-676.

Hill, R.M., Pettit, J.W., Lewinsohn, P.M., Seeley, J.R., \& Klein, D.N. (2014). Escalation to major depressive disorder among adolescents with subthreshold depressive symptoms: Evidence of distinct subgroups at risk. Journal of Affective Disorders, 158, 133-138.

Huan, V.S., See, Y.L., Ang, R.P., \& Har, C.W. (2008). The impact of adolescent concerns on their academic stress. Educational Review, 60, 161-178.

Keller, M.B., Lavori, P.W., Wunder, J., Beardslee, W.R., Schwartz, C.E., \& Roth, J. (1992). Chronic course of anxiety disorders in children and adolescents. Journal of the American Academy of Child and Adolescent Psychiatry, 31, 595-599.

Kessler, R.C., Berglund, P., Demler, O., Jin, R., \& Walters, E.E. (2005). Lifetime prevalence and age-of-onset distributions of DSM-IV disorders in the National Comorbidity Study replication. Archives of General Psychiatry, 62, 593-602.

Kim-Cohen, J., Caspi, A., Moffitt, T.E., Harrington, H., Milne, B.J., \& Poulton, R. (2003). Prior juvenile diagnoses in adults with mental disorder: Developmental follow-back of a prospectivelongitudinal cohort. Archives of General Psychiatry, 60, 709-717.

Last, C.G., Perrin, S., Hersen, M., \& Kazdin, A.E. (1996). A prospective study of childhood anxiety disorders. Journal of the American Academy of Child and Adolescent Psychiatry, 35, 1502-1510.

Lazarus, R.S., \& Folkman, S. (1984). Stress, appraisal, and coping. Nueva York: Springer.

Lin, H.J.. \& Yussoff, M.S.B. (2013). Psychological distress, sources of stress and coping strategy in high school students. International Medical Journal, 20, 672 .
Lonigan, C.J., \& Phillips, B.M. (2001). Temperamental influences on the development of anxiety disorders. En M.W. Vasey y M.D. Dadds (Eds.), The developmental psychopathology of anxiety (pp. 60-91). Nueva York: Oxford University Press.

Mangunkusumo, R., Moorman, P., van den Berg-de Ruiter, A., van der Lei, J., de Koning, H., \& Raat, H. (2005). Internet-administered adolescent questionnaires comparison of web-based versus paper-and-pencil selfadministered questionnaire compared with a paper version in a randomized study. Journal of Adolescent Health, 36, 70.e1-70.e6.

Miller, G.A., \& Chapman, J.P. (2001). Misunderstanding analysis of covariance. Journal of Abnormal Psychology, 110, 40-48.

Muris, P., \& Ollendick, T.H. (2005). The role of temperament in the etiology of child psychopathology. Clinical Child and Family Psychology Review, 8, 271289.

Nadkarni, A., \& Hofmann, S.G. (2012). Why do people use Facebook? Personality and Individual Differences, 52, 243-249.

Newman, D.L., Moffitt, T.E., Caspi, A., Magdol, L., Silva, P.A., \& Stanton, W.R. (1996). Psychiatric disorder in a birth cohort of young adults: Prevalence, comorbidity, clinical significance, and new case incidence from ages 11 to 21. Journal of Consulting and Clinical Psychology, 64, 552-562.

Oliva, A., Jiménez, J.M., Parra, A., \& Sánchez-Queija, I. (2008). Acontecimientos vitales estresantes, resiliencia y ajuste adolescente. Revista de Psicopatología y Psicología Clínica, 13, 53-62.

Putwain, D.W. (2011). How is examination stress experienced by secondary students preparing for their General Certificate of Secondary Education examinations and how can it be explained? International Journal of Qualitative Studies in Education, 24, 717-731.

Rothbart, M.K. (2007). Temperament, development, and personality. Current Directions in Psychological Science, 16, 207-212.

Ryan, T., \& Xenos, S. (2011). Who uses Facebook? An investigation into the relationship between the Big Five, shyness, narcissism, loneliness and Facebook usage. Computers in Human Behavior, 27, 1658-1664.

Salmera-Aro, K., \& Tynkkynen, L. (2012). Gendered pathways in school burnout among adolescents. Journal of Adolescence, 35, 929-939.

Sandín, B., Valiente, R.M., y Chorot, P. (2009). RCADS: evaluación de los síntomas de los trastornos de ansiedad y depresión en niños y adolescentes. Revista de Psicopatología y Psicología Clínica, 14, 193-206.

Schulenberg, S.E.. \& Yutrzenka, B. A. (1999). The equivalence of computerized and paper-and-pencil psychological instruments: Implications for measures 
of negative affect. Behavior Research Methods, Instruments y Computers, 31, 315-321.

Schutz, P.A., Benson, J., \& Decuir-Gunby, J.T. (2008). Approach/Avoidance motives, test emotions, and emotional regulation related to testing. Anxiety, Stress, \& Coping: An International Journal, 21, 3, 263-281.

Silverman, W.K., \& Ollendick, T.H. (2005). Evidencebased assessment of anxiety and its disorders in children and adolescents. Journal of Clinical Child \& Adolescent Psychology, 34, 380-411.

Snyder, J., Bullard, L., Wagener, A., Leong, P.K., Snyder, J., \& Jenkins, M. (2009). Childhood anxiety and depressive symptoms: trajectories, relationship, and association with subsequent depression. Journal of Clinical Child and Adolescent Psychology, 38, 837849.

Spielberger, C. (1989). Inventario de Ansiedad Estado Rasgo para niños y adolescentes (STAIC). Madrid: TEA Ediciones.

Sportel, B.E., Nauta, M.H., de Hullu, E., \& de Jong, P.J. (2013). Predicting internalizing symptoms over a two year period by BIS, FFFS and attentional control. Personality and Individual Differences, 54, 236-240.

Torrubia, R., García-Carrillo, M., Àvila, C., Caseras, X., \& Grande, I. (2008). A junior questionnaire for the measurement of Sensitivity to Punishment and
Sensitivity to Reward. Manuscrito no publicado, Universidad Autónoma de Barcelona.

Tortella-Feliú, M., Balle, M., \& Sesé, A. (2010). Relationships between negative affectivity, emotion regulation, anxiety, and depressive symptoms in adolescents as examined through structural equation modeling. Journal of Anxiety Disorders, 24, 686-693.

Van Oort, F.V.A., Greaves-Lord, K., Verhulst, F.C., Ormel, J., \& Huizink, A.C. (2009). The developmental course of anxiety symptoms during adolescence: The TRAILS study. Journal of Child Psychology and Psychiatry, 50, 1209-1217.

Weems, C.F. (2008). Developmental trajectories of childhood anxiety: Identifying continuity and change in anxious emotion. Developmental Review, 28, 488-502.

Wyrick, D.L., \& Bond, B. (2011) Reducing sensitive survey response bias in research on adolescents: A comparison of web-based and paper-and-pencil administration. American Journal of Health Promotion, 25, 349-352.

Zeidner, M. (1998). Test anxiety: The state of the art. Nueva York: Plenum Press.

Zimmerman, M., \& Martinez, J.H. (2011). Web-based assessment of depression in patients treated in clinical practice: Reliability, validity, and patient acceptance. The Journal of Clinical Psychiatry, 73, 333-338. 